\title{
Colonoscopic Treatment of a Fecaloma at the Anastomotic Site after Colectomy
}

\author{
Yuko Homma Toshiki Mimura Ai Sadatomo Koji Koinuma \\ Hisanaga Horie Alan Kawarai Lefor Naohiro Sata
}

Division of Gastroenterological, General and Transplant Surgery, Department of Surgery, Jichi Medical University, Shimotsuke, Japan

\section{Keywords}

Fecaloma · Functional end-to-end anastomosis · Colectomy · Colonoscopy · Polyethylene glycol

\begin{abstract}
Fecalomas most commonly occur in constipated patients and are rarely reported after colectomy. A 55-year-old Japanese female presented with a fecaloma after colectomy, which was impacted at a functional end-to-end anastomosis (FEEA) site. Four and a half years ago, she underwent sigmoidectomy for colon cancer. A follow-up computed tomography (CT) scan revealed an $11 \mathrm{~cm}$ incidental fecaloma. The patient was advised to undergo surgery, but she desired nonoperative management because of minimal symptoms, and was referred to our institution. On the day of admission (day 1), mechanical fragmentation of the fecaloma was attempted during the first colonoscopy. Although a large block of stool was evacuated after a second colonoscopic fragmentation on day 8, the third colonoscopy on day 21 and CT scan on day 22 showed no significant change in the fecaloma. Frequent colonoscopic fragmentation was performed, with a fourth, fifth, and sixth colonoscopy on days 24, 29, and 31, respectively. After the size reduction was confirmed at the sixth colonoscopy, the patient was discharged home on day 34. The fecaloma completely resolved after the seventh colonoscopic fragmentation on day 44. Sixteen months after treatment, there is no evidence of recurrent fecaloma. According to the literature, risk factors for fecaloma after colectomy include female gender, left-side colonic anastomosis, and FEEA. FEEA might not be recommended for anastomoses in the left colon, particularly in female patients, to avoid this complication. Colonoscopic fragmentation is recommended for fecalomas at an anastomotic site after colectomy in patients without an absolute indication for surgery.
\end{abstract}




\section{Introduction}

Fecaloma is "a hard, laminated, and calcified fecal mass," which most commonly occurs in the rectum or sigmoid colon [1]. Many cases of fecaloma have been described in patients with chronic constipation in association with psychiatric, Chagas', or Hirschsprung's diseases $[2,3]$. Fecaloma has rarely been reported in patients after colectomy without underlying conditions. However, in the era of laparoscopic surgery with stapled anastomoses, fecaloma has been reported as a rare but important complication at the anastomotic site, particularly at the site of a functional end-to-end anastomosis (FEEA). We report a patient with an $11 \mathrm{~cm}$ fecaloma impacted at a stapled FEEA site after sigmoidectomy, successfully treated with colonoscopic fragmentation and oral polyethylene glycol (PEG).

\section{Case Presentation}

A 55-year-old Japanese female presented with a fecaloma impacted at the anastomotic site after sigmoidectomy. Four and half years prior to referral, she underwent sigmoidectomy reconstructed with a stapled FEEA for an advanced sigmoid colon cancer (T3N1M0, Stage IIIB) at another hospital. The operation was performed through an open laparotomy because she underwent hysterectomy and left salpingo-oophorectomy for myoma uteri and a left ovarian cyst simultaneously.

Although she did not have constipation preoperatively, she developed chronic constipation with decreased bowel frequency after resection, treated with magnesium oxide (330 $\mathrm{mg}, 3$ times a day) together with stimulant laxatives (Sennoside $24 \mathrm{mg}$ ) as needed. Her bowel movement frequency, however, was once weekly on this regimen. The patient described occasional abdominal pain and bloating, but no symptoms of difficult evacuation or sensation of incomplete evacuation. Four and a half years postoperatively, a computed tomography (CT) scan was performed for the follow-up. While no recurrence or metastases were found, an incidental $11 \mathrm{~cm}$ fecaloma was found at the anastomotic site in the sigmoid colon (Fig. 1a). She was advised to undergo an emergent operation, although she had only mild abdominal distension. She wanted nonoperative therapy and was referred to our hospital, where specialized management is available for severe constipation through our service for functional anorectal and bowel disorders.

For 1 week before presentation, she took only liquids by mouth to avoid aggravation of the fecaloma, which she feared might necessitate an emergent surgery. At presentation, she was in no acute distress and denied feeling incomplete evacuation or other gastrointestinal symptoms except infrequent bowel movements. On physical examination, her abdomen was slightly distended and a large, firm, smooth, nontender, and slightly mobile mass was palpable in the lower abdomen. On digital rectal examination, the rectum was empty, but a hard mass was palpable in Douglas' pouch through the rectal wall. Laboratory tests showed no remarkable abnormalities. Abdominal X-ray showed a large, round opacity filling the pelvis (Fig. 1b), consistent with the fecaloma seen on the recent CT scan.

The patient was admitted for nonoperative therapy and further evaluation (Table 1). On hospital day 1, the first colonoscopy was performed without bowel preparation, which showed a fecaloma at the site of the previous sigmoid colon anastomosis (Fig. 2a), but the endoscope could not be advanced proximally. Fluoroscopy with Gastrografin during colonoscopy demonstrated its shape, while Gastrografin easily passed proximally (Fig. 2b). Mechanical fragmentation of the fecaloma was attempted with biopsy forceps under colonoscopic monitoring with some success. To follow the fecaloma, abdominal ultrasonography was performed on day 2, which showed a round acoustic shadow $11.6 \mathrm{~cm}$ in maximum

\section{Karger'}



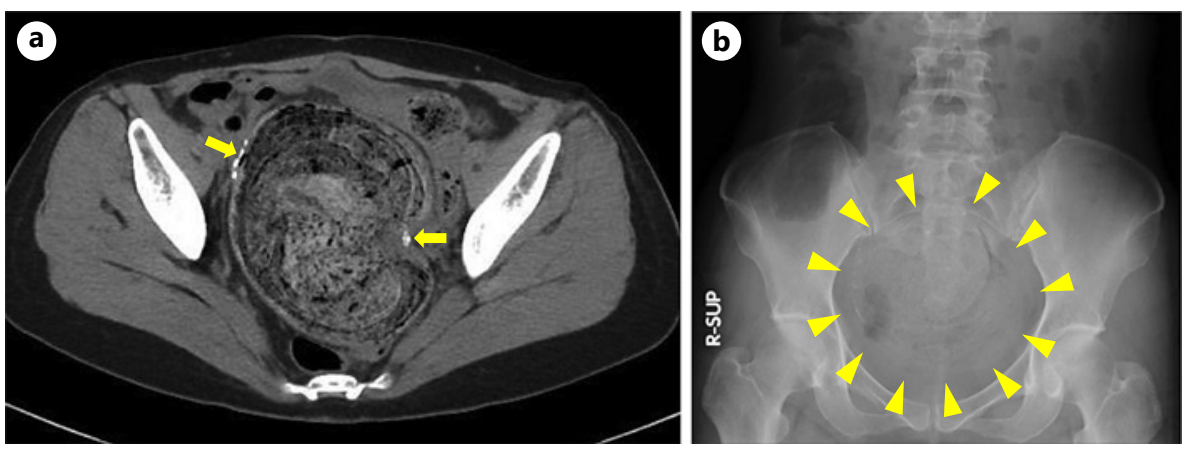

Fig. 1. a Computed tomography scan at the previous hospital shows an 11-cm fecaloma, impacted at the anastomotic site in the sigmoid colon. Arrows indicate the staples at the functional end-to-end anastomosis. b Abdominal X-ray on admission shows a large, round opacity filling the pelvis, suggesting a fecaloma, marked by arrowheads.

Table 1. Clinical course

\begin{tabular}{|c|c|}
\hline Hospital day & Treatment progress \\
\hline 1 & $\begin{array}{l}\text { Admission } \\
\text { 1st colonoscopy \& fluoroscopy with Gastrografin: mechanical fragmentation } \\
\text { was performed }\end{array}$ \\
\hline 2 & $\begin{array}{l}\text { Fecaloma: } 11.6 \mathrm{~cm} \text { in diameter on ultrasonography } \\
\text { Started fluid diet and oral intake of PEG } 120 \mathrm{~mL} / \text { day }\end{array}$ \\
\hline 6 & PEG increased up to $360 \mathrm{~mL} /$ day \\
\hline 8 & 2nd colonoscopy: mechanical fragmentation \\
\hline 9 & Evacuated a large block of stool \\
\hline 17 & Normal diet with low dietary fiber \\
\hline 21 & $\begin{array}{l}\text { Full bowel preparation with oral PEG of 2,000 mL } \\
\text { 3rd colonoscopy: mechanical fragmentation }\end{array}$ \\
\hline 22 & CT scan: no significant decrease of fecaloma $(9 \times 7 \mathrm{~cm})$ \\
\hline 24 & 4th colonoscopy: mechanical fragmentation \\
\hline 29 & 5th colonoscopy: mechanical fragmentation \\
\hline 30 & Fecaloma decreased down to $5.8 \mathrm{~cm}$ in diameter on ultrasonography \\
\hline 31 & 6th colonoscopy: mechanical fragmentation \& PEG injection into the fecaloma \\
\hline 34 & Discharged \\
\hline 44 & 7th colonoscopy: mechanical fragmentation \\
\hline 55 & Evacuated a large stool \\
\hline 58 & 8th colonoscopy: no stool in the entire colon including the anastomosis site \\
\hline \multicolumn{2}{|c|}{ Follow-up after treatment } \\
\hline 4 months later & CT scan: no fecaloma at the colonic anastomosis \\
\hline 13 months later & 9th colonoscopy: no stool in the entire colon including the anastomosis site \\
\hline 16 months later & No recurrence of fecaloma without symptoms of constipation, on oral PEG \\
\hline
\end{tabular}

diameter. On the same day, an oral diet limited to liquids was started because the colonoscopy suggested a minimal risk of developing colonic obstruction. Oral administration of PEG 120 $\mathrm{mL} /$ day was gradually increased to $360 \mathrm{~mL}$ /day on day 6 . On day 8 , the second colonoscopy 

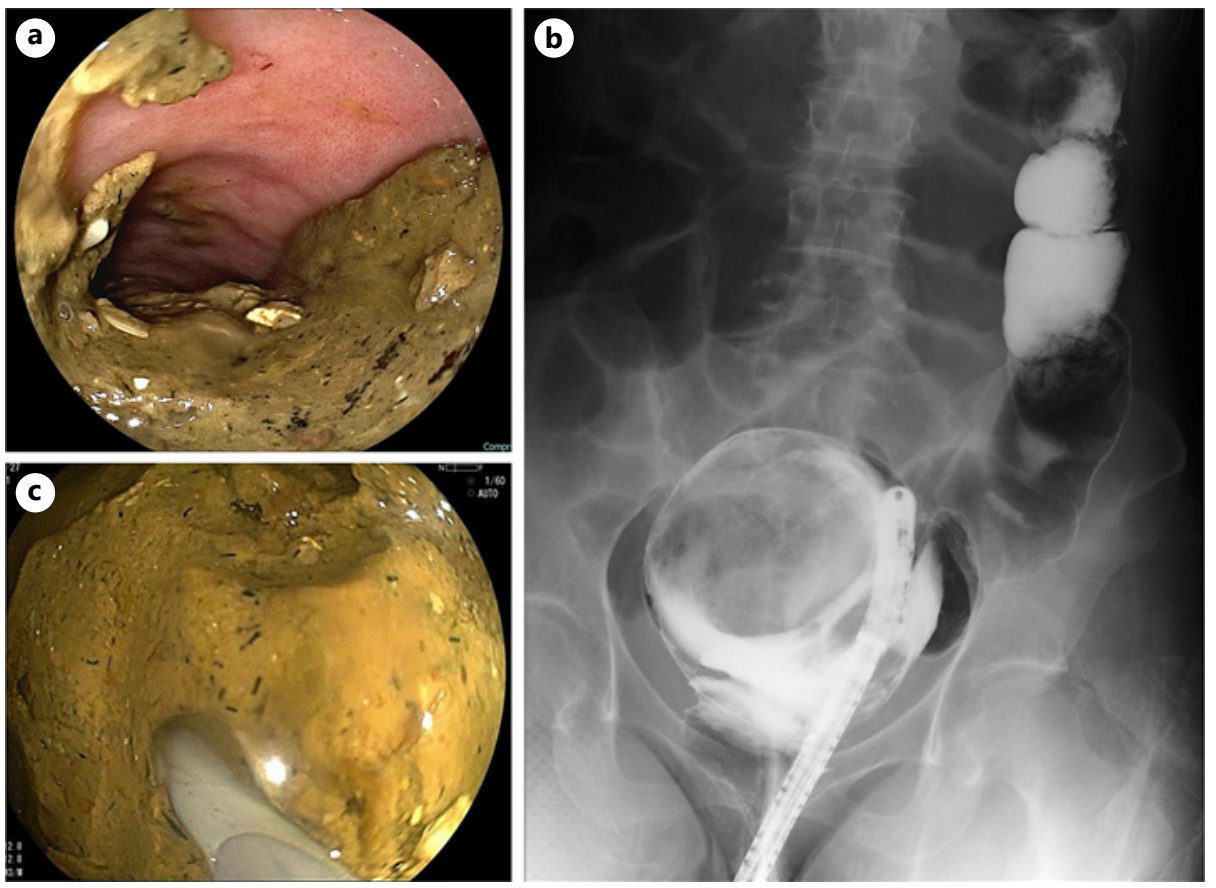

Fig. 2. a Colonoscopy on day 1 shows a fecaloma at the anastomotic site in the sigmoid colon. b Fluoroscopy with Gastrografin under the colonoscopy demonstrates the shape of the fecaloma, and the Gastrografin flows proximally into the descending colon. c At the sixth colonoscopy on day 31, polyethylene glycol liquid was injected into the fecaloma and fragmented with biopsy forceps.

was performed without bowel preparation because of concern for the risk of developing colonic obstruction or perforation if she underwent complete bowel preparation with 2,000 $\mathrm{mL}$ of oral PEG. Mechanical fragmentation of the fecaloma was again attempted with some success. The patient spontaneously evacuated a large block of stool on day 9, which supported a continued use of oral PEG, and therefore, the oral intake was gradually increased to a normal low fiber diet on day 17 . While receiving laxatives, she passed loose or watery stool 2-7 times per day.

The third colonoscopy was performed on day 21 after bowel preparation with 2,000 mL of oral PEG, which showed no gross abnormalities in the colon other than the fecaloma. Although we anticipated a significant reduction in the size of the fecaloma, the size appeared similar, and mechanical fragmentation with biopsy forceps was repeated. The palpable abdominal mass remained similar in size, and the second CT scan on day 22 showed no significant decrease in the size of the fecaloma.

Without a significant change in size, we decided to treat the fecaloma by frequent colonoscopic fragmentation sessions. The fourth and fifth colonoscopic fragmentation sessions were performed on days 24 and 29 with some decrease in the size of the fecaloma, confirmed on palpation and abdominal ultrasonography, which showed a decrease in its maximum diameter from $11.6 \mathrm{~cm}$ on day 2 to $5.8 \mathrm{~cm}$ on day 30. At the sixth colonoscopy on day 31, PEG liquid was injected into the fecaloma with fragmentation using biopsy forceps (Fig. 2c). Due to a decrease in the size of the fecaloma confirmed by colonoscopy, she was discharged home on day 34 .

During the 7th colonoscopy, performed as an outpatient on day 44, mechanical fragmentation was repeated because the fecaloma remained, although it had further decreased in size. When she visited for the 8 th colonoscopy on day 58 , she told us that she had passed a large 

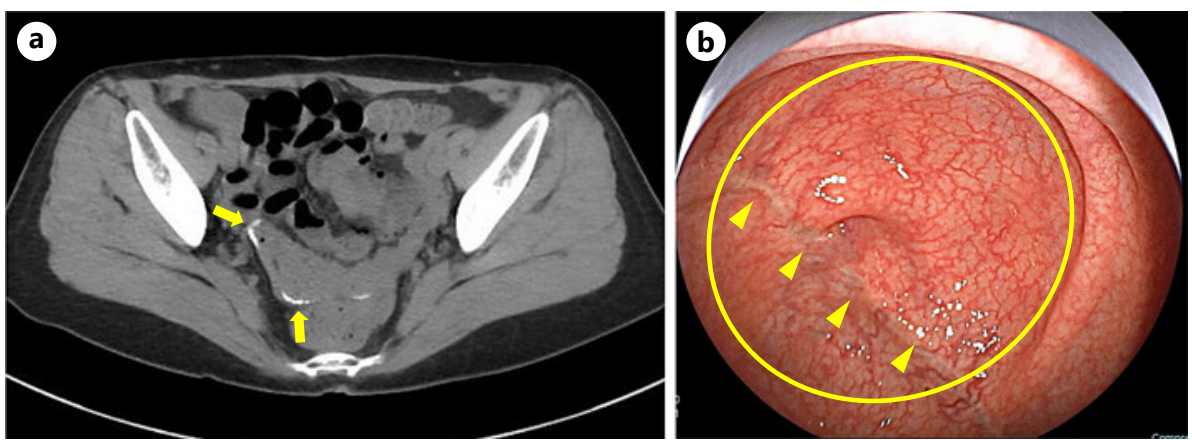

Fig. 3. a Computed tomography scan 4 months after treatment shows no fecaloma at the colonic anastomotic site. Arrows indicate the staples at the site of the functional end to end anastomosis. $\mathbf{b}$ The ninth colonoscopy 13 months after treatment shows no fecaloma at the colonic anastomosis. Some dilatation was observed at the anastomosis. Arrowheads indicate the staple line of the anastomosis, and a circle indicates the dilatation area.

stool on day 55. Colonoscopy revealed that the fecaloma was no longer in the colon. The patient was extremely satisfied with the success of nonoperative therapy.

Four months later, a follow-up CT scan showed no fecaloma at the anastomotic site (Fig. 3a). Thirteen months after treatment, a 9th colonoscopy was performed because the entire colon was not clearly observed at the third colonoscopy, and showed no abnormalities in the entire colon, and no evidence of a fecaloma. Some dilatation was observed at the anastomosis (Fig. 3b). Sixteen months after treatment, the patient has no symptoms of constipation, continuing oral administration of PEG, $120 \mathrm{~mL} 3$ times a day $(360 \mathrm{~mL} /$ day $)$ with bowel movement frequency of once or twice per day of semisolid stool.

\section{Discussion/Conclusion}

An 11-cm fecaloma developed at the site of an FEEA four and a half years after sigmoidectomy in this patient. This is a rare but important complication in the era of mechanical anastomoses. The fecaloma was successfully treated with 7 sessions of colonoscopic fragmentation and oral administration of PEG, obviating the need for surgery, which had been initially recommended at the previous hospital. Sixteen months after treatment, the fecaloma has not recurred, with no symptoms of constipation, and still taking oral PEG.

Fecaloma is defined as "a hard, laminated, and calcified fecal mass," which most commonly occurs in the rectum and sigmoid colon [1]. Many cases of fecaloma have been described in patients suffering from chronic constipation in association with psychiatric, Chagas', or Hirschsprung's diseases [2,3]. It has rarely been reported in patients after colectomy without underlying conditions. However, in the era of laparoscopic surgery with mechanically performed anastomoses, several cases of fecaloma have been reported as rare but important complications at the anastomotic site, particularly after FEEA.

To the best of our knowledge, 6 patients with fecaloma have been reported, which developed at the anastomotic site after colorectal surgery [4-9] (Table 2). The English language literature was searched with the key words ("fecaloma" or "fecalith" or "fecal impaction" or "blind loop syndrome" or "blind pouch syndrome" or "stercoral") and ("surgery" or "anastomosis" or "colonic surgery" or "functional end to end" or "side-to-side" or "side-toend" or "hand sewn" or "double stapling" or "DST") on PubMed for papers between January 2001 and February 2021. Of 657 studies found, only 1 case was identified as a fecaloma, 


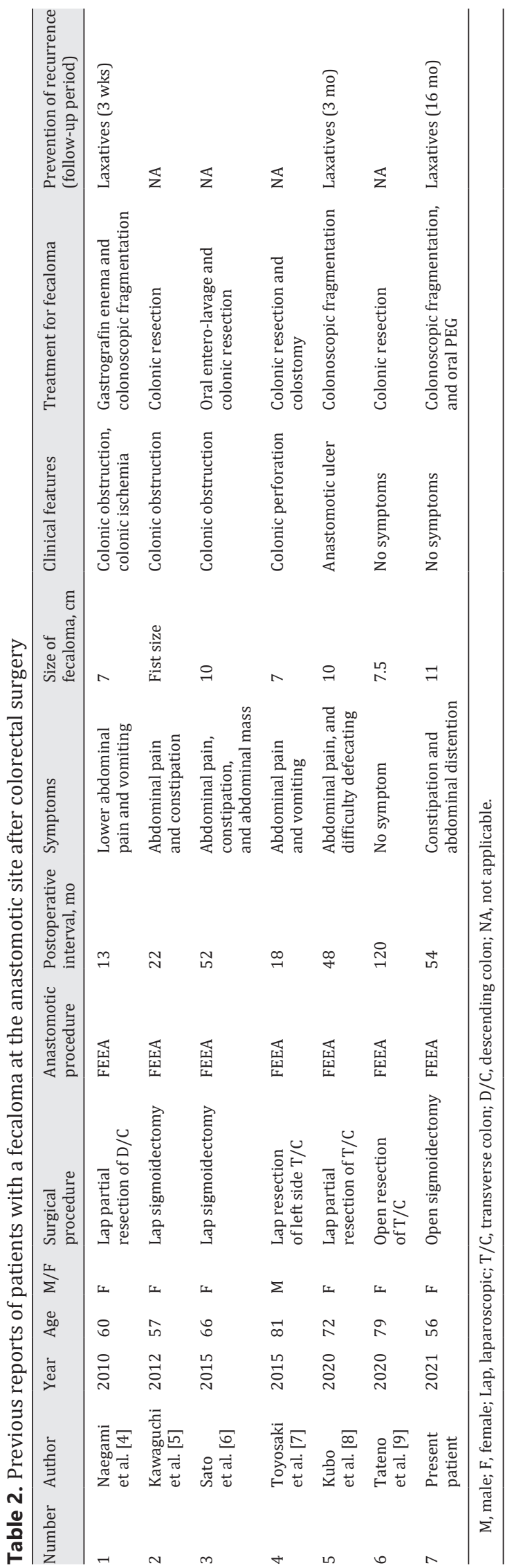


which developed at an anastomotic site after colorectal surgery [8]. The Japanese language literature was also searched with the same key words on Japan Medical Abstracts Society during the same period. Of 394 possible papers, 5 cases were identified as a fecaloma, which developed at the anastomotic site after colorectal surgery.

All 7 patients, including the present patient, were reported after 2010. The age of the patients ranges between 56 and 81 years, and 6/7 were female. Four patients underwent left colectomy, while 3 patients underwent transverse colectomy. All anastomotic procedures were FEEA. These characteristics suggest that risk factors for fecaloma include a female gender, a left-side colonic anastomosis, and FEEA. Females are susceptible to chronic constipation, and the stool is generally firmer on the left side than on the right side of the colon. Among all anastomotic procedures identified in the literature, fecaloma developed only at the site of an FEEA. As mechanisms of a fecaloma develop at FEEA, we speculate that the colon at the site of an FEEA tends to become extremely large compared to the proximal and distal colonic diameters, creating a kind of diverticulum, which can cause the stasis of feces, resulting in a fecaloma. Toyosaki et al. [7] and Tateno et al. [9] advocated the same mechanisms. In the present patient, some dilatation was actually observed at the anastomosis as shown in Fig. 3b. The risk factor of left-side colonic anastomoses for fecaloma might be associated with the larger diameter of a left colonic anastomosis, as surgeons tend to make the anastomosis larger for left-sided anastomoses than for the right-sided anastomoses because of harder and larger stool in the left colon.

The FEEA was first reported in 1968 by Steichen [10] and has become popular for anastomoses after right hemicolectomy and small bowel resections because it saves time, increases the anastomotic diameter, and makes it easier to anastomose lumens of different diameters [11]. For left colonic anastomoses, it was performed as early as 2003 [12] and has become more frequently used in the era of laparoscopic surgery with stapled anastomoses [13]. Considering the risk factors for fecaloma above, however, it might be better to avoid an FEEA for anastomoses of left colon, particularly in female patients.

As shown in Table 2, the interval between colectomy and development of a fecaloma ranged between 13 and 120 months. Fecaloma can be a late complication after the FEEA. All patients but one had symptoms including abdominal pain, constipation, vomiting, abdominal mass, difficulty defecating, and abdominal distention. The size of reported fecalomas ranges from 7 to $11 \mathrm{~cm}$ with the present patient having the largest fecaloma reported to date. Regarding clinical features, 3 patients presented with colonic obstruction and 1 patient had a perforation, requiring an emergent surgery.

Surgery with colonic resection was performed in 4 patients $[5-7,9]$, while nonoperative therapy with colonoscopic fragmentation was carried out in $3[4,8]$ (Table 2). Among the 4 patients who underwent surgery, Kawaguchi et al. [5] and Toyosaki et al. [7] performed a colonic resection without any preceding treatment. Tateno et al. [9] unsuccessfully used colonoscopic fragmentation of the fecaloma before the surgery. Sato et al. [6] also tried colonoscopic fragmentation and then treated the fecal mass causing colonic obstruction with oral entero-lavage. After the fecal mass was completely evacuated, they further resected the emptied colon including the anastomotic region because it was abnormally dilated. Although their treatment was eventually successful, there may have been an increased risk of perforation because oral entero-lavage is generally contraindicated with colonic obstruction. In the present patient, we performed colonoscopy without preparation before confirming the absence of colonic obstruction.

Of 3 patients treated nonoperatively, Naegami et al. [4] removed an impacted fecal mass at the anastomotic site with a Gastrografin enema, followed by only 1 session of colonoscopic fragmentation. Kubo et al. [8] performed colonoscopic fragmentation 4 times using snares and alligator forceps over 4 consecutive days. In the present patient, we performed colonoscopic

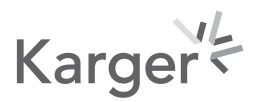


fragmentation 7 times over 55 days. We expected that the fecaloma could be treated only with oral PEG after the second colonoscopic treatment. If we had initially performed colonoscopic fragmentation more frequently, treatment might have been completed earlier.

Indications for surgery include colonic perforation and obstruction. Otherwise, nonoperative therapy such as colonoscopic fragmentation is recommended, although it could take a longer time for complete resolution in some patients. Since the present patient was eager to avoid surgery, we tried to treat her nonoperatively, which took about 2 months. Before starting nonoperative therapy, therefore, we must explain that it might take longer than surgery, and that oral laxatives may be required even after treatment. To some extent, the choice of treatment might depend on patients' preference.

To prevent recurrence of a fecaloma, some laxatives might have to be continued in patients who are treated nonoperatively. As shown in Table 2, all 3 patients took laxatives after nonoperative therapy. In the present patient, the fecaloma has not recurred 16 months after treatment, and she has no symptoms of constipation on oral PEG. We used PEG not only for treatment of the fecaloma but also for prevention because it is a highly effective and welltolerated laxative for the treatment of severe constipation, particularly for fecal impaction [14].

In conclusion, an 11-cm fecaloma was successfully treated with 7 sessions of colonoscopic fragmentation and oral PEG. Fecaloma is a rare but important late complication after colectomy in the era of mechanical anastomoses. From the literature, the risk factors for fecaloma include female gender, left-side colonic anastomosis, and FEEA. It might be better to avoid FEEA for anastomoses in the left colon, particularly in female patients, to reduce the risk of this complication. Colonoscopic fragmentation is recommended for a fecaloma at the anastomotic site after colectomy in patients without an indication for surgery.

\section{Statement of Ethics}

Ethics approval is not required in accordance with our national guidelines. Written informed consent was obtained from the patient for publication of this case report and any accompanying images.

\section{Conflict of Interest Statement}

All authors declare no conflicts of interests regarding the publication of this paper.

\section{Funding Sources}

None of the authors have received any funding regarding this paper.

\section{Author Contributions}

All authors in this manuscript contributed to the interpretation of data, and drafting and writing of this manuscript. Y.H., T.M., A.S., K.K., and H.H. were engaged in patient's care in her hospital course including endoscopy under the supervision of T.M., K.K., H.H., and N.S. T.M. and A.L. contributed to drafting and writing of the manuscript and interpretation of data. All authors have read and approved this manuscript for publication. 
Homma et al.: Colonoscopic Treatment of Fecaloma

\section{Data Availability Statement}

All data generated or analyzed during this study are included in this article. Further inquiries can be directed to the corresponding author.

\section{References}

1 Rajagopal A, Martin J. Giant fecaloma with idiopathic sigmoid megacolon: report of a case and review of the literature. Dis Colon Rectum. 2002;45(6):833-5.

2 Mushtaq M, Shah MA, Malik AA, Wani AK, Thakur N, Parray FQ. Giant fecaloma causing small bowel obstruction: case report and review of the literature. Bull Emerg Trauma. 2015;3(2):70-2.

3 Currò G, Lazzara C, Latteri S, Bartolotta M, Navarra G. Supergiant fecaloma as manifestation of chronic constipation. G Chir. 2017;38(1):53-4.

4 Naegami N, Satou M, Watabe S, Saitou T, Ishido Y, Yamada M. A case of fecal ileus caused by fecal impaction in the intestine at the anal side of a functional end-to-end anastomotic site. J Jpn Surg Assoc. 2010;71(5):121621.

5 Kawaguchi T, Hiratsuka T, Izumi K, Aramaki M. A case of obstruction caused by accumulation of faces at the site of a functional end-to-end anastomosis after an operation for colon cancer. J Clin Surg. 2012;67(9): 1202-4.

6 Sato Y, Kuroki Y, Kudo H, Hayashi H. A case of blind loop syndrome in the functional end-to-end anastomotic site of laparoscopic surgery. J Jpn Surg Assoc. 2015;76(11):2735-9.

7 Toyosaki R, Kanmura M, Oyama T, Mita T, Sato R, Kitazono M. A case of perforation caused by accumulation of feces at the site of a functional end-to-end anastomosis after laparoscopy assisted transverse colectomy. J Jpn Surg Assoc. 2015;76(12):3029-33.

8 Kubo K, Suzuoki M, Kimura N, Maiya N, Matsuda S, Tsuda M, et al. Blind pouch syndrome-associated enterolithiasis successfully treated with colonoscopy. Case Rep Gastroenterol. 2020;14(3):527-33.

9 Tateno Y, Ishii M, Hayashi T, Hataji K, Tezuka T, Katsuragawa H. A case of a giant enterolith formed at a functional end-to-end anastomosis requiring surgical removal. J Jpn Surg Assoc. 2020;81(11):2285-9.

10 Steichen FM. The use of staplers in anatomical side-to-side and functional end-to-end enteroanastomoses. Surgery. 1968;64(5):948-53.

11 Naito M, Miura H, Nakamura T, Sato T, Yamanashi T, Tsutsui A, et al. Sutureless functional end-to-end anastomosis using a linear stapler with polyglycolic acid felt for intestinal anastomoses. Ann Med Surg. 2017;17: 50-3.

12 Sakamoto N, Ogata J, Yoneda K, Mori M, Hasue K, Wada T, et al. Functional end-to-end anastomosis in openabdominal surgery for colon cancer: based on experience with 153 cases. J Jpn Coll Surg. 2003;28(2):251-6.

13 Ohya H, Watanabe J, Suwa H, Suwa Y, Ishibe A, Masui H, et al. Incidence and risk factors for fluorescence abnormalities on near-infrared imaging using indocyanine green in stapled functional end-to-end anastomosis in laparoscopic colectomy. Int J Colorectal Dis. 2020;35(11):2011-8.

14 Chen CC, Su MY, Tung SY, Chang FY, Wong JM, Geraint M. Evaluation of polyethylene glycol plus electrolytes in the treatment of severe constipation and faecal impaction in adults. Curr Med Res Opin. 2005;21(10):1595602. 